\title{
Sesquiterpenos e hidrocarbonetos dos frutos de Xylopia emarginata (Annonaceae)
}

\author{
Isabel C. Moreira ${ }^{1}$, Nídia F. Roque ${ }^{1}$, Karla Contini', João Henrique G. Lago ${ }^{2 *}$ \\ ${ }^{I}$ Departamento de Química Fundamental, Instituto de Química, Universidade de São Paulo, \\ 05599-970, São Paulo, SP, Brasil, \\ ${ }^{2}$ Departamento de Química, Centro de Ciências e Humanidades, Universidade Presbiteriana Mackenzie, \\ 01302-907, São Paulo, SP, Brasl
}

\begin{abstract}
RESUMO: O extrato hexânico dos frutos de Xylopia emarginata foi particionado entre hexano e $\mathrm{MeOH} / \mathrm{H}_{2} \mathrm{O}$. A fase hidroalcoólica foi submetida à separação cromatográfica fornecendo quatro sesquiterpenos: óxido de cariofileno, espatulenol, 1 $\beta, 6 \alpha$-diidroxi-4(15)-eudesmeno e 4-hidroxi-1,15peróxieudesmano. A fase hexânica foi fracionada através de cromatografia em coluna fornecendo dois hidrocarbonetos (nonadecano e 1-nonadeceno) e uma cetona alifática (hentriacontan-16-ona). As estruturas dos compostos isolados foram estabelecidas através de análise espectroscópica, principalmente RMN e EM.
\end{abstract}

Unitermos: Xylopia emarginata, sesquiterpenos, hidrocarbonetos.

\begin{abstract}
Sesquiterpenes and hydrocarbons from Xylopia emarginata (Annonaceae) fruits". The hexane extract from Xylopia emarginata fruits was partitioned between hexane and $\mathrm{MeOH} / \mathrm{H}_{2} \mathrm{O}$. The hydro-alcoholic phase was submitted to chromatographic separation to afford four sesquiterpenes: caryophyllene oxide, spathulenol, 1及,6 $\alpha$-dihydroxy-4(15)-eudesmene and 4hydroxy-1,15-peroxy-eudesmane. The hexane phase was fractioned in column chromatography to afford two hydrocarbons (nonadecane and 1-nonadecene) and one aliphatic ketone (hentriacontan16-one). The structures of the isolated compounds were established by spectral data analysis, mainly NMR and MS.
\end{abstract}

Keywords: Xylopia emarginata, sesquiterpenes, hydrocarbons.

\section{INTRODUÇÃO}

Estudos químicos de espécies vegetais da família Annonaceae têm mostrado o acúmulo de diferentes metabólitos secundários com importantes atividades farmacológicas tais como citotóxica (flavanonas), antitumoral (diterpenos, alcalóides), bactericida (alcalóides) e antifúngica (terpenos) (Martins, 1996; Moreira et al., 2003a). O gênero Xylopia, pertencente à família Annonaceae, é constituído por aproximadamente 160 espécies, das quais diversas são usadas na medicina popular brasileira, tais como $X$. aethiopica para o tratamento de bronquite e disenteria, $X$. aromatica como agente diurético e para edema de pele, $X$. sericeae como antiinflamatório e $X$. cayennensis para transtornos cardiovasculares (Moreira, 1999; Macedo; Ferreira, 2004; Nascimento et al., 2006).

A espécie Xylopia emarginata Mart. é vulgarmente conhecida como pindaíba-reta, pindaíbad'água, pindaíba-do-brejo, embira-preta, pindaíba, pindaúba e pindaibuna as quais variam de acordo com a região em que a espécie é encontrada. Consiste em uma árvore de porte médio, de 10 a 20 metros de altura com um tronco de 30 a $40 \mathrm{~cm}$ de diâmetro. Ocorre em lugares brejosos e em matas ciliares, principalmente nos estados da Bahia, Minas Gerais, Goiás, Mato Grosso do Sul e São Paulo. Sua floração inicia-se em julho, tendo maior abundância de outubro a fevereiro ao passo que a frutificação ocorre nos meses de julho a agosto (Lorenzi, 1992).

Estudos fitoquímicos com essa espécie vegetal descreveram a ocorrência de diterpenos, sesquiterpenos, alcalóides e esteróides nos frutos (Vilegas et al., 1991; Moreira et al., 2003b) esteróides, sesquiterpenos, diterpenos e flavonóides nas folhas (Moreira et al., 2003b), adutos diterpênicos dos galhos (Moreira et al., 2006) além de monoterpenos e sesquiterpenos no óleo essencial das folhas (Lago et al., 2005; Brochini et al. 1999). No entanto, apesar desta espécie ter sido estudada exaustivamente do ponto de vista fitoquímico, poucos foram os trabalhos onde se verificou o acúmulo de metabólitos de baixa polaridade. Assim, no presente estudo, o extrato em hexano dos frutos de X. emarginata foi submetido a fracionamento cromatográfico de onde foram isolados quatro sesquiterpenos: óxido de cariofileno (1), espatulenol (2), 1 $\beta, 6 \alpha$-diidroxi-4(15)eudesmeno (3) e 4-hidroxi-1,15-peróxieudesmano (4), dois hidrocarbonetos: nonadecano (5) e 1-nonadeceno 
(6) e uma cetona alifática: hentriacontan-16-ona (7). As estruturas das substâncias isoladas foram definidas através da análise dos espectros de $\mathrm{RMN}$ de ${ }^{1} \mathrm{H}$ e de ${ }^{13} \mathrm{C}$ além de espectrometria de massas seguido da comparação com dados descritos na literatura. Das substâncias isoladas 4 é inédita como produto natural enquanto que 5, 6 e 7 estão sendo descritos pela primeira vez na espécie em estudo.

\section{MATERIAL E MÉTODOS}

\section{Equipamentos}

Os espectros de RMN foram obtidos em espectrômetro Bruker AC-200 operando a $200 \mathrm{MHz}$ para o núcleo do hidrogênio e a $50 \mathrm{MHz}$ para o núcleo do carbono-13. As amostras foram solubilizadas em $\mathrm{CDCl}_{3}$ (Aldrich) contendo 1\% de tetrametilsilano (TMS) como padrão interno. Os espectros de massas foram registrados em um espectrômetro INCOS 50 FinniganMat-quadrupole operando por impacto eletrônico a 70 eV contando com biblioteca de espectros NIST. Nas separações cromatográficas em coluna aberta foi utilizado gel de sílica 60 (Merck) enquanto que nas análises com camada fina foi utilizado gel de sílica $60 \mathrm{PF}_{254}$ (Merck) $(0.25 \mathrm{~mm})$ revelado com sulfato cérico seguido de aquecimento por 5 minutos.

\section{Material vegetal}

Os frutos de $X$. emarginata $(380 \mathrm{~g})$ foram coletados no campus da Universidade Federal do Mato Grosso do Sul, Campo Grande, MS, em agosto de 1995. A identificação da planta foi realizada pelo Dr. Renato Mello Silva do Instituto de Biociências da Universidade de São Paulo (IB-USP). Uma exsicata do material está depositada no Herbário do IB-USP (SFP), sob número 101500 .

\section{Extração e isolamento dos constituintes químicos}

Os frutos foram secados a $60{ }^{\circ} \mathrm{C}$ por 48 horas e moídos, fornecendo $380 \mathrm{~g}$ de material. Esse foi submetido à extração com hexano até esgotamento fornecendo $22 \mathrm{~g}$ do extrato hexânico.

$\mathrm{O}$ extrato hexânico foi submetido à partição com $\mathrm{MeOH} / \mathrm{H}_{2} \mathrm{O}$ (95:5) fornecendo $14 \mathrm{~g}$ da fase hexânica e $8 \mathrm{~g}$ da fase hidroalcoólica. Esta última, após evaporação do solvente seguido de metilação com diazometano, foi submetida à cromatografia em gel de sílica utilizando uma mistura gradiente de $\mathrm{CH}_{2} \mathrm{Cl}_{2}$ em hexano. Esse procedimento forneceu 71 frações $(100 \mathrm{~mL}$ cada) que foram reunidas em 12 grupos após análise por CCDC. O grupo 5 foi submetido a cromatografia em camada delgada preparativa $\left(\mathrm{CH}_{2} \mathrm{Cl}_{2}\right.$ puro) fornecendo $10 \mathrm{mg}$ de 1. Uma mistura das substâncias $\mathbf{1}$ e $\mathbf{2}$ foi obtida do grupo 7 $(23 \mathrm{mg}$ ) ao passo que 2 foi isolado do grupo 8 (20 mg). O grupo 9 foi submetido à cromatografia em camada delgada preparativa $\left(\mathrm{CH}_{2} \mathrm{Cl}_{2}: \mathrm{MeOH}\right.$ 9:1) fornecendo $15 \mathrm{mg}$ de 3 . A substância $4(6 \mathrm{mg})$ foi isolada após purificação por cromatografia em coluna de gel de sílica $\left(\mathrm{CH}_{2} \mathrm{Cl}_{2}: \mathrm{MeOH}\right.$ 85:15) do grupo 11.

A fase hexânica foi submetida à cromatografia em coluna de gel de sílica utilizando uma mistura gradiente de $\mathrm{CH}_{2} \mathrm{Cl}_{2}$ em hexano e posteriormente $\mathrm{MeOH}$ em $\mathrm{CH}_{2} \mathrm{Cl}_{2}$ fornecendo 95 frações $(100 \mathrm{~mL}$ cada) que foram reunidas em oito grupos após análise por CCDC. Uma mistura das substâncias 5 e 6 (19 mg) foi obtida do grupo 3 após recristalização com MeOH. O grupo 7 (24 mg) mostrou-se constituído pela substância 7.

Óxido de cariofileno (1): Óleo incolor. EM $\mathrm{m} / \mathrm{z}$ (int. rel. \%): $220[\mathrm{M}]^{+}(2), 161(7), 147(6), 133(8)$, 121(26), 119(16), 109(39), 105(34), 93(72), 91(76), 81(37), 79(100), 69(49), 67(43), 55(35); RMN ${ }^{1} \mathrm{H}$ $\left(\mathrm{CDCl}_{3}\right): \delta 4,97$ (H-15a), 4,86 (H-15b), 2,90 (dd, $J=10,6$ e 4,3 Hz, H-1), 2,61 (t, $J=9,4 \mathrm{~Hz}, \mathrm{H}-5), 1,20$ (s, H-15), 1,01 (s, H-12), 1,00 (s, H-13); RMN ${ }^{13} \mathrm{C}\left(\mathrm{CDCl}_{3}\right): \delta 152,1$ (C-4), 113,0 (C-14), 64,0 (C-1), 60,0 (C-10), 50,9 (C-7), 49,0 (C-5), 40,0 (C-6), 39,4 (C-9), 34, 2 (C-11), 30,4 (C2), 30,2 (C-13), 30,0 (C-3), 27,4 (C-8), 21,8 (C-12), 17,2 (C-15).

Espatulenol (2): Óleo incolor. EM $\mathrm{m} / \mathrm{z}$ (int. rel. \%): $220[\mathrm{M}]^{+}(1), 204(11), 189(22), 175(10), 161(49)$, 147(25), 133(30), 121(39), 105(75), 91(76), 79(80), 67(100), 55(82); $\mathrm{RMN}{ }^{1} \mathrm{H}\left(\mathrm{CDCl}_{3}\right): \delta 4,69$ (s, H-15a), 4,66 (s, H-15b), 2,39 (dd, $J=14,1$ e $6,3 \mathrm{~Hz}, \mathrm{H}-1$ ), 1,28 (s, H-14), 1,06 (s, H-13), 1,05 (s, H-12), 0,72 (ddd, $J=$ $11,2 ; 9,5$ e $6,1 \mathrm{~Hz}, \mathrm{H}-7), 0,46$ (dd, $J=11,2$ e 9,5 Hz, H6); $\mathrm{RMN}{ }^{13} \mathrm{C}\left(\mathrm{CDCl}_{3}\right): \delta 153,4(\mathrm{C}-10), 106,2(\mathrm{C}-15), 80,9$ (C-4), 54,3 (C-1), 53,3 (C-5), 41,7 (C-3), 38,8 (C-9), 29,9 (C-6), 28,6 (C-12), 27,4 (C-7), 26,7 (C-2), 26,0 (C-14), 24,8 (C-8), 20,2 (C-11), 16,2 (C-13).

$1 \beta, 6 \alpha$-Diidroxi-4(15)-eudesmeno (3): Óleo incolor. EM $\mathrm{m} / \mathrm{z}$ (int. rel. \%): 238(17), 220(100), 205(28), 201(7), 177(89); $\mathrm{RMN}^{1} \mathrm{H}\left(\mathrm{CDCl}_{3}\right): \delta 5,02(\mathrm{~d}, J=1,0 \mathrm{~Hz}$, H-15a), 4,74 (d, $J=1,0 \mathrm{~Hz}, \mathrm{H}-15 \mathrm{~b}), 3,72$ (t, $J=10,0 \mathrm{~Hz}$, H-6), 3,42 (dd, $J=11,3$ e 4,7 Hz, H-1), 0,95 (d, $J=7,0$ $\mathrm{Hz}, \mathrm{H}-13), 0,87$ (d, $J=7,0 \mathrm{~Hz}, \mathrm{H}-12), 0,71$ (s, H-14); $\mathrm{RMN}{ }^{13} \mathrm{C}\left(\mathrm{CDCl}_{3}\right)$ : $\delta 146,2(\mathrm{C}-4), 107,8(\mathrm{C}-15), 79,0(\mathrm{C}-$ 1), 67,0 (C-6), 55,9 (C-5), 49,3 (C-7), 41,7 (C-10), 36,3 (C-9), 35,1 (C-3), 31,9 (C-2), 26,0 (C-11), 21,1 (C-12), 18,2 (C-8), 16,2 (C-13), 11,6 (C-14).

4-Hidroxi-1,15-peróxieudesmano (4): Óleo incolor. EM $m / z$ (int. rel. \%): $254[\mathrm{M}]^{+}(10), 222(36)$, 221(13), 220(10), 207(19), 205(13), 180(31), 165(32), 163(33), 137(70), 109(95), 135(52), 91(66), 81(98), 55(100); $\mathrm{RMN}^{1} \mathrm{H}\left(\mathrm{CDCl}_{3}\right): \delta 3,67$ (d, $\left.J=9,2 \mathrm{~Hz}, \mathrm{H}-15 \mathrm{a}\right)$, $3,54$ (d, $J=9,2 \mathrm{~Hz}, \mathrm{H}-15 \mathrm{~b}), 3,33$ (dd, $J=11,0$ e 4,2 Hz, H-1), 1,00 (s, H-14), 0,92 (d, $J=6,9$ Hz, H-13), 0,86 (d, $J=6,9 \mathrm{~Hz}, \mathrm{H}-12)$; $\mathrm{RMN}{ }^{13} \mathrm{C}\left(\mathrm{CDCl}_{3}\right): \delta 80,5(\mathrm{C}-1), 80,3$ (C-15), 75,6 (C-4), 57,5 (C-5), 51,1 (C-7), 39,7 (C-9), 33,2 (C-2), 32,2 (C-10), 28,0 (C-3 e C-6), 27,8 (C-11), 22,2 (C-8), 20,7 (C-13), 18,5 (C-12), 12,8 (C-14).

Nonadecano (5): Sólido branco amorfo. RMN ${ }^{1} \mathrm{H}\left(\mathrm{CDCl}_{3}\right): \delta 1,21$ (sl, H-2 - H-18), 0,87 (t, $J=6,7 \mathrm{~Hz}$, 


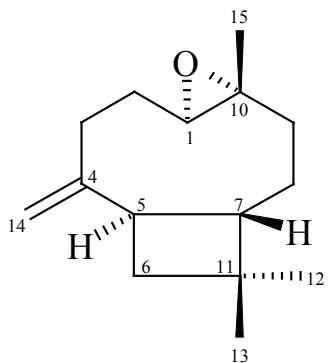

1<smiles>CCCCCC</smiles>

5<smiles>C=C1CCC2C([C@H]3[C@@H]1CC[C@@]3(C)O)C2(C)C</smiles>

2<smiles>C=C1CCC(O)[C@]2(C)CCC(C(C)C)[C@H](O)[C@@H]12</smiles>

3<smiles>CC(C)C1CCC2(C)C3CC[C@H](O)[C@]2(C1)OO3</smiles>

4<smiles>C=CCCC</smiles>

6<smiles>CCCCC(C)C(=O)O</smiles>

7

Figura 1. Sesquiterpenos e hidrocarbonetos isolados dos frutos de X. emarginata.

H-1 e H-19).

1-Nonadeceno (6): Sólido branco amorfo. RMN ${ }^{1} \mathrm{H}\left(\mathrm{CDCl}_{3}\right): \delta 5,79$ - 5,71 (m, H-2), 4,98 - 4,91 (m, H-1), 2,03 (m, H-3), 1,21 (sl, H-4 - H-18), 0,87 (t, $J=6,7$ Hz, H-19).

Hentriacontan-16-ona (7): Sólido branco amorfo. EM m/z (int. rel. \%): $450[\mathrm{M}]^{+}$(1), 239(100), 254(19), 194(13), 71(70). IV (KBr) $v_{\max }: 2918,2849$, $1703 \mathrm{~cm}^{-1}$; RMN ${ }^{1} \mathrm{H}\left(\mathrm{CDCl}_{3}\right): \delta 2,37(\mathrm{t}, J=6,7 \mathrm{~Hz}, 2 \mathrm{H})$, $1,57(\mathrm{~m}, 2 \mathrm{H}), 1,18(\mathrm{sl}), 0,87(\mathrm{t}, J=6,7 \mathrm{~Hz}, 3 \mathrm{H})$; RMN ${ }^{13} \mathrm{C}$ $\left(\mathrm{CDCl}_{3}\right): \delta 211,9$ (C-16), 42,8 (C-15 e C-17), 31,9 (C-16 e C-18), 31,9 - 22,6 (C-19 a C-30 e C-2 a C-14), 14,2 (C-1 e C-31).

\section{RESULTADOS E DISCUSSÃO}

As estruturas dos sesquiterpenos óxido de cariofileno (1), espatulenol (2) e 1 $\beta, 6 \alpha$-diidroxi-4(15)eudesmeno (3) foram definidas com base nos espectros de $\mathrm{RMN}$ de ${ }^{1} \mathrm{H}$, de ${ }^{13} \mathrm{C}$ e de massas e por comparação com dados descritos na literatura.

$\mathrm{O}$ espectro de $\mathrm{RMN}$ de ${ }^{13} \mathrm{C}$ de $\mathbf{1}$ mostra quinze sinais, sendo dois relativos a carbonos de ligação dupla exocíclica $(\delta 152,1$ e 113,0$)$ e dois referentes a carbonos carbinólicos $(\delta 64,0$ e 60,0$)$, os quais são característicos de grupos epóxido. Esses dados, associados àqueles obtidos do espectro de massas, o qual mostrou o pico do íon molecular em $\mathrm{m} / \mathrm{z} 220$, são indicativos da estrutura do óxido de cariofileno, a qual foi confirmada após comparação com dados da literatura (Heymann et al., 1994). No espectro de $\mathrm{RMN}$ de ${ }^{1} \mathrm{H}$ de 2 foram observados dois sinais em campo alto em $\delta 0,72(\mathrm{ddd}, J=11,2 ; 9,5$ e $6,1 \mathrm{~Hz})$ e $0,46(\mathrm{dd}, J=11,2 \mathrm{e} 9,5 \mathrm{~Hz})$ os quais foram atribuídos a H-6 e H-7 de sesquiterpenos de esqueleto aromadendrano. Adicionalmente, a presença de dois singletos em $\delta 4,66(1 \mathrm{H})$ e 4,69 $(1 \mathrm{H})$, característicos de hidrogênio de dupla exocíclica além de três singletos em $\delta$ $1,05(3 \mathrm{H}), 1,06(3 \mathrm{H})$ e $1,28(3 \mathrm{H})$, referentes a três grupos metílicos, sendo o último ligado a carbono oxigenado, sugeriu a estrutura do espatulenol, a qual foi confirmada após análise dos espectros de RMN de ${ }^{13} \mathrm{C}$ e EM seguido de comparação com dados da literatura (Brochini; Roque, 2000; Adams, 2001). O espectro de RMN de ${ }^{1} \mathrm{H}$ de 3 mostra dois dubletos em $\delta 0,95(J=7,0 \mathrm{~Hz})$ e $0,87(J=$ $7,0 \mathrm{~Hz}$ ), característicos de grupo isopropílico. A presença de uma ligação dupla exocíclica foi sugerida pelos sinais em $\delta 5,02(\mathrm{~d}, J=1,0 \mathrm{~Hz})$ e 4,74 (d, $J=1,0 \mathrm{~Hz}$ ) enquanto que a presença de dois grupos hidroxílicos na molécula deveu-se aos sinais em $\delta 3,72(\mathrm{t}, J=10,0 \mathrm{~Hz})$ e 3,42 (dd, $J=11,3$ e $4,7 \mathrm{~Hz}$ ). A análise por RMN de ${ }^{13} \mathrm{C}$ confirmou tais grupamentos devido aos sinais de carbonos $\mathrm{sp}^{2} \mathrm{em}$ $\delta 146,2(\mathrm{C})$ e 107,8 $\left(\mathrm{CH}_{2}\right)$ e oximetínicos em $\delta 79,0$ e 67,0. Finalmente, após verificação das multiplicidades dos carbonos pelo experimento DEPT $135^{\circ}$, foi possível sugerir a estrutura de 3 como $1 \beta, 6 \alpha$-diidroxi-4(15)eudesmeno, a qual foi confirmada após comparação com dados da literatura (González et al, 1989; Gutierrez et al., 1988). O espectro de $\mathrm{RMN}$ de ${ }^{13} \mathrm{C}$ de 4 mostra 15 sinais, indicativos da ocorrência de um sesquiterpeno. Do mesmo modo que em $\mathbf{3}$, a multiplicidade dos sinais observados no espectro DEPT $135^{\circ}$, associado aos sinais observados no espectro de $\mathrm{RMN}$ de ${ }^{1} \mathrm{H}$ o qual mostra dentre outros, dois dubletos em $\delta 0,86(J=6,9$ $\mathrm{Hz}, 3 \mathrm{H})$ e $\delta 0,92(J=6,9 \mathrm{~Hz}, 3 \mathrm{H})$, característicos um grupo isopropílico e um singleto em $\delta 1,00(3 \mathrm{H})$, relativo a grupo metílico, são indicativos de um sesquiterpeno 
de esqueleto eudesmano. Ainda no espectro de RMN de ${ }^{13} \mathrm{C}$ foram observados três carbonos carbinólicos em $\delta$ $80,5(\mathrm{CH}), 80,3\left(\mathrm{CH}_{2}\right)$ e 75,6 (C) além de três grupos metílicos em $\delta 20,718,5$ e 12,8. Uma vez que o esqueleto eudesmano prevê a presença de quatro grupos metílicos, foi sugerido que $\mathrm{C}-15$ encontra-se oxidado visto que os sinais de C-12, C-13 e C-14 são similares aos de 3. Uma vez que foram observados, no espectro de $\mathrm{RMN}$ de ${ }^{1} \mathrm{H}$, dois dubletos atribuídos aos H-15 em $\delta$ 3,67 $(J=9,2$ $\mathrm{Hz})$ e 3,54 (d, $J=9,2 \mathrm{~Hz})$, conclui-se que C-4 também é carbinólico. Finalmente, através da análise por EM, onde o pico do íon-molecular foi observado em $\mathrm{m} / \mathrm{z} 254$, referente a fórmula molecular $\mathrm{C}_{15} \mathrm{H}_{26} \mathrm{O}_{3}$, foi proposta a ocorrência de um endoperóxido, entre C-1 e C-15. Desta forma, a estrutura de $\mathbf{4}$ foi definida como 4-hidróxi-1,15peróxieudesmano, cuja ocorrência é inédita como produto natural. $\mathrm{O}$ espectro de $\mathrm{RMN}$ de ${ }^{1} \mathrm{H}$ da mistura contendo as substâncias 5 e $\mathbf{6}$ mostrou sinais referentes à hidrogênios ligados a carbonos $\mathrm{sp}^{2}$ em $\delta$ 5,79 - 5,71 (m), 4,98 - 4,91 $(\mathrm{m})$, de hidrogênios ligados a carbonos em cadeia longa em $\delta 1,21(\mathrm{sl})$ além de grupo metílico em $\delta 0,87(\mathrm{t}, J$ $=6,7 \mathrm{~Hz}$ ). Desta forma, foi proposta a ocorrência de dois compostos cuja diferenciação consistia apenas a presença da ligação dupla terminal. Assim, após análise por CG-EM e comparação com os espectros disponíveis na espectroteca do aparelho, seguido da co-injeção com padrões autênticos, os mesmos foram identificados como nonadecano (5) e 1-nonadeceno (6). O espectro de $\mathrm{RMN}$ de ${ }^{1} \mathrm{H}$ de 7 mostra, além dos sinais em $\delta 1,18$ (sl), e 0,87 (t, $J=6,7 \mathrm{~Hz}$ ) atribuídos a hidrogênios metilênicos e metílicos de cadeia longa, um tripleto em $\delta 2,37(J=6,7 \mathrm{~Hz}, 2 \mathrm{H})$, relativo aos hidrogênios ligados ao carbono $\alpha$-carbonílico. A presença desse grupamento foi confirmada pela observação, no espectro de RMN de ${ }^{13} \mathrm{C}$, de um sinal em $\delta 211,9(\mathrm{C}=\mathrm{O})$ e da banda de estiramento do grupo carbonila em $1703 \mathrm{~cm}^{-1}$, no espectro de infravermelho. O EM mostrou o pico do íon molecular em $m / z 450$, compatível com a fórmula $\mathrm{C}_{31} \mathrm{H}_{62} \mathrm{O}$, e o pico relativo ao rearranjo de McLafferty em $\mathrm{m} / z$ 254, o qual possibilitou o posicionamento do grupo carbonílico em C-16. Finalmente, a estrutura de 7 foi definida como a da hentriacontan-16-ona.

Apesar de vários trabalhos com o gênero Xylopia descreverem a ocorrência de terpenos e alcalóides, poucos são aqueles que investigam a composição das frações apolares. Neste trabalho, além de se detectar quatro sesquiterpenos (1 - 4), foram identificados três derivados alifáticos $(\mathbf{5}$ - 7). A produção e acúmulo de sesquiterpenos vêm sendo descrita em várias espécies do gênero sendo o espatulenol (2) de ocorrência irrestrita (Moreira, 1999).

\section{AGRADECIMENTOS}

Os autores agradecem a CNPq pelo apoio financeiro. KC e ICM agradecem a FAPESP pelas bolsas de estudo concedidas.

\section{REFERÊNCIAS}

Adams RP 2001. Identification of essential oil components by gas chromatography/quadrupole mass spectrometry. Illinois: USA, Allured Publ. Corp., Carol Stream.

Brochini CB, Nunez CV, Moreira IC, Roque NF, Chaves MH, Martins D 1999. Identificação de componentes de óleos voláteis: análise espectroscópica de misturas de sesquiterpenos. Quim Nova 22: 37-40.

Brochini CB, Roque NF 2000. Two new cneorubin related diterpenes from the leaves of Guarea guidonia (Meliaceae). J Braz Chem Soc 11: 361-364.

GonzalezAG, Bermejo-Barrera J, Yanes AC, DiazJG, RodriguezPerez EM 1989. Chromenes and benzofurans from Ageratina glechonophylla Phytochemistry 28: 25202522.

Gutierrez AB, Herz W 1988. Guaianolides and other constituents of Helianthus microcephalus. Phytochemistry 27: 2225-2228.

Heymann H, Tezuka Y, Kikuchi T, Supriyatna S 1994. Constituents of Sindora sumatrana Miq. Isolation and NMR spectral analysis of sesquiterpenes from the dried pods. Chem Pharm Bull 42: 138-140.

Lago JHG, Reis AA, Martins D, Cruz FG, Roque NF 2005. Composition of the leaf oil of Xylopia emarginata Mart. (Annonaceae). J Essent Oil Res 17: 622-623.

Lorenzi H, Árvores Brasileiras: Manual de identificação e cultivo de plantas arbóreas nativas do Brasil, Nova Odessa/SP, Editora Plantarum, 1992, p. 18 e 19.

Macedo M, Ferreira AR 2004. Plantas medicinais usadas para tratamentos dermatológicos, em comunidades da Bacia do Alto Paraguai, Mato Grosso. Rev Bras Farmacogn 14(Supl. 1): 40-44.

Martins D 1996. Alcalóides, flavonóides e terpenóides de Xylopia aromatica. São Paulo, 134p. Tese de Doutorado Instituto de Química, Universidade de São Paulo.

Moreira IC 1999. Estudo químico de Xylopia emarginata e Xylopia brasiliensis. São Paulo, 203p. Tese de Doutorado - Instituto de Química, Universidade de São Paulo.

Moreira IC, Lago JHG, Young MCM, Roque NF 2003a. Antifungal aromadendrane sesquiterpenoids from the leaves of Xylopia brasiliensis. J Braz Chem Soc 14: 828-831.

Moreira IC, Lago JHG, Roque NF 2003b. Alkaloid, flavonoids and terpenoids from leaves and fruits of Xylopia emarginata (Annonaceae). Bioch Syst Ecol 31: 535537.

Moreira IC, Roque NF, Lago JHG 2006. Diterpene adducts from branches of Xylopia emarginata. Bioch Syst Ecol 34: 833-837.

Nascimento AA, Ribeiro EAN, Oliveira JM, Medeiros FA, Silva MS, Medeiros IA 2006. Cardiovascular effects induced by the hydroalcoholic extract of the stem of Xylopia cayennensis in rats. Rev Bras Farmacogn 16: 17-21.

Vilegas W, Felício JD, Roque NF, Gottlieb HE 1991. Diterpenic adducts from Xylopia species. Phytochemistry 30: 1869-1872. 